\title{
Design-insights for Devising Persuasive IoT Devices for Sustainability in the Workplace
}

\author{
Diego Casado-Mansilla*†, Anne M. irizar*†, Pablo Garaizar ${ }^{\dagger}$, and Diego López-de-Ipiña* ${ }^{* \dagger}$ \\ *DeustoTech - Fundación Deusto, Avda. Universidades, 24, 48007, Bilbao \\ ${ }^{\dagger}$ Facultad Ingeniería, Universidad de Deusto, Avda. Universidades, 24, 48007, Bilbao \\ \{dcasado, ane.irizar, garaizar, dipina\}@deusto.es
}

\begin{abstract}
Sustainable HCI scholars have shown a growing interest in augmenting with technology everyday devices for promoting energy conservation behaviors in different contexts of use. The workspace has not been sufficiently explored as space where everyday appliances and work equipment might be converted into persuasive devices which raise eco-awareness. In this paper, we present the design of persuasive IoT devices that help to promote energy-efficient behaviour change in the workplace. This approach issued by applying Grounded Theory analysis over qualitative data collected at ten focus groups sessions after running a one year study where participants were treated to different persuasive strategies. With these data, five design categories emerged to be taken into account in the conception phase when devising tangible objects that aim to form pro-environmental behaviour change at workplaces. Our design-insights are also derived from an evaluation of the literature on ambient devices that encourage energy-efficient practices.
\end{abstract}

\section{INTRODUCTION}

In the recent years, the Human Computer-Interaction (HCI) community started to increase its attention in sustainability issues because environmental quality strongly depends on human behaviour. A large body of previous research has evidenced the value of persuasive energy feedback on behaviour change [11][18][21]. Persuasion for sustainability has its roots in the application of Fogg's framework for "computers as persuasive technologies" to the topic of environmental sustainability [10]. In fact, most of the research works on sustainability have based their theoretical rationale on this model [15].

This paper focuses in a single but a widespread environmental issue which is reducing the energy footprint at workplace. Brynjarsdttir et al. [5] found that more than a half of the papers reviewed in their analysis tackled energy related topics. Similarly, Pierce and Paulos [18] stated that energy consumption was the primary focus on sustainable HCI works after reviewing 51 papers on the field. More relevant to our interests was to understand that the analysis carried out on this topic revealed that there is a growing interest on augmenting legacy or new everyday devices with persuasive technology to make them help people to form or enhance eco-minded behaviours. However, due to the diversity of sustainable actions to improve and the users' diversity, it is hard to find a set of guidelines tailored for the specific purpose that the designer desires. Hence, with this piece of research, we seek to offer an ensemble of designinsights that researchers on the area of sustainability, and specifically in energy awareness through IoT, might follow when approaching the design of persuasive systems.

\section{BACKGROUND}

In the existing literature, we found non-negligible body of research where everyday devices were augmented to make users aware about their energy consumption by using physical metaphors and aesthetic interfaces. In this Section, these works are reviewed and then evaluated based on ten common metrics applied when designing systems for behaviour change [22].

The review starts with the Power-aware Cord, an electrical power-strip in which the cord was designed to expose the electricity through ambient light. The bigger the energy drawn by the power-strip, the brighter the cord is [11]. Waterbot provides ambient feedback about water usage in a kitchen's sink through visual and auditory remainders [3]. Similarly, 'Show-me' displays the amount of water that is being used during the shower through a LED strip assembled to the shower's stick [14]. Stroppy Kettle is an augmented appliance that aimed to break user's kettle overfill behaviour applying barriers to goal-attainment and punishment [8]. Watt-lite [13] and Energy Aware clock [4] are two works that aim to explore tangible data and non-obtrusive interaction to reduce energy consumption. Social-coffee maker [6] is an instrumented everyday appliance that predicts when to subtly interact through visualizations with its users to make them operate the device in a more intelligent and energy efficient fashion. Thieme et al. [21] devised BinCam, a social persuasive rubbish-bin with a built-in camera to motivate tenants to adopt recycling habits and reduce food waste. Interactive living plants were designed by Huh et al. [12]. The authors created a robotic analogue of a plant that mimics photo-tropic behaviour.

There are also some products commercially available in this area. Wattson from DIY Kyoto ${ }^{1}$ shows the overall electricity use in numbers and colours. Another product that can be found in the market is the Ambient Orb². It

\footnotetext{
${ }^{1}$ http://inhabitat.com/diy-kyotos-wattson/

${ }^{2} \mathrm{http} / / /$ ambientdevices.myshopify.com/products/energy-orb
} 
is a frosted-glass ball that illuminates a varying degree of colours to represent critical peak demand conditions on the smart grid. Finally, the Nest Thermostat ${ }^{3}$ is a smart device that aims to learn user's heating and cooling habits to help optimise scheduling and power usage. The devices reward the users with a Leaf if they setup the temperature according to the Nest recommendations to save energy.

Previously reviewed works can be observed in the collage of Figure 1.

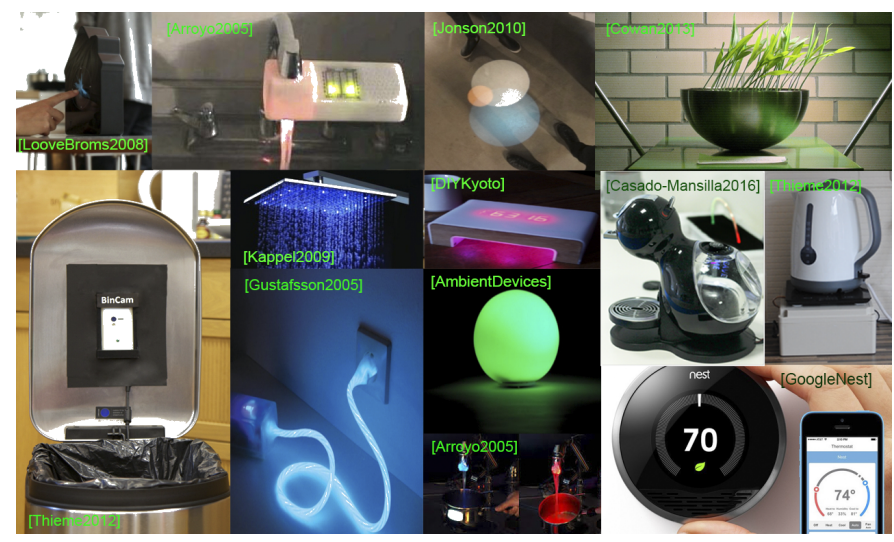

Figure 1. Augmented objects found in the literature which were designed for promoting sustainable behaviour change through tangible or ambient interaction.

\section{A. Evaluation of the State of the Art}

Table I summarises the evaluation criteria for each of the reviewed artifacts. The ten metrics used for evaluating the state of the art [22] are described below: 1) Rational vs Emotional: the former defines the use of providing rational or pragmatic information as a strategy for behavior change (e.g providing energy measurements). The latter refers to the use of artistic or emotional characteristics within the intention in order to provoke desired feelings in the user to change its behaviour; 2) Easy vs. Difficult to Use: The former evaluates the facility of use of an augmented device as a principle to generate a change in attitude or behaviour. The latter provided the degree of obstrusiveness or limitation of use that is built-in in the system to make difficult or avoid undesired actions or affordances; 3) Direct route vs. Peripheral route of persuasion: the former uses elements that make the user generate a self-concious reasoning that affects the behavior in a direct way. The latter defines the degree in which the design provides cues through a secondary route (e.g. subtle tips or advices) for changing the intended behaviour; 4) Positive vs. Negative reinforcement: the former measures the degree of use of positive and motivational concepts for user engagement (e.g. prising). The latter evaluates whether the proposed strategies are used to prevent or remove undesired user's

\footnotetext{
${ }^{3}$ https://nest.com/thermostat/meet-nest-thermostat/
}

Table I

AUGMENTED OBJECTS EVALUATED THROUGH TEN LENSES FOR PRO-ENVIRONMENTAL BEHAVIOUR CHANGE. THE REVIEWED PROJECTS TAGGED WITH AN ASTERISK WERE TESTED IN WORK ENVIRONMENTS.

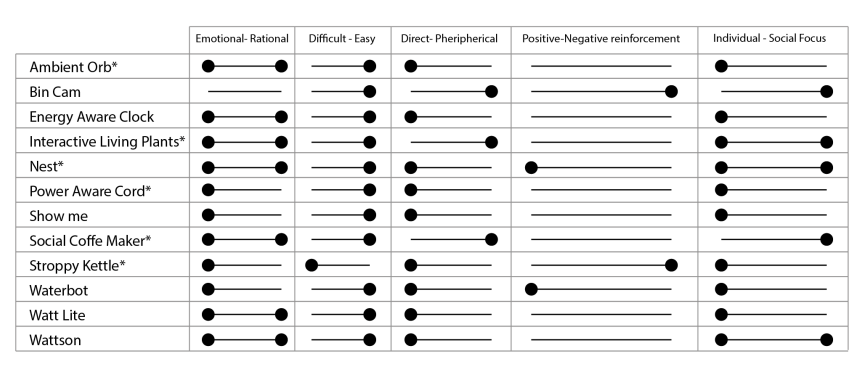

behavior; 7) Individual vs. Social focus: the former refers to the use of personal and subjective norms or beliefs to generate sustainable behavior. The latter uses the influence of social norms as a strategy for forming or sustain the target behaviour.

In a glimpse, it can be observed that the majority of the works reviewed devised everyday things taking into account the emotional bond that final users may create with the object or the objective that these pursue. Furthermore, reviewed works were mainly focused on the individual, easy to use in order to facilitate the adoption of the targeted behaviour and, finally, there is an unanimity of works that decided to apply the central or direct route for providing persuasive cues. We took into consideration these ten metrics when we approached the design of the persuasive devices that we used to conduct the longitudinal study that is explained in the next section.

\section{Procedure}

We carried out an experimental intervention in which we recorded the energy consumption of several electrical coffeemachines in fifteen different workplaces distributed between two big cities of Spain (Madrid and Bilbao). The experiment lasted one year. More than eighty people that worked in any of these fifteen recruited working spaces participated in the experiment. They were all recruited following a snowball procedure and their participation was voluntary (we raffled an energy monitoring equipment among participants that completed the study at its conclusion). The intervention was designed to test the effectiveness of the persuasive techniques in the mid and long-term because of some scholars raised their concern about the feasibility of persuasion to maintain the target behaviour throughout the time [9][15].

We decided to instrument capsule-based coffee machines to become the main actors to convey persuasive messages. The reason why we selected these appliances were that (1) they are pretty common in work environments and are 
element of shared use ${ }^{4}$; (2) they consume large amounts of energy compared to other work appliances such as monitors or laptops. The coffee machines were appliances of common use in these semi-public spaces. At the beginning of the study, the fifteen coffee-makers were augmented with energy monitoring technology to obtain their energy consumption baseline (this baseline lasted 8 months). After this period, we augmented the appliances with interactive technologies. The study that we conducted was conceived to follow a between-group design approach. Thus, three different strategies to cope with energy inefficiency were tested among the participant groups. The three strategies, that can be observed in Figure 2 are described hereafter: 1) Delayed feedback: participants were provided with a Web site to track their energy consumption associated to the appliance (i.e. self-monitoring and rational information); 2) Automation: the coffee makers were modified to autonomously switch the appliances off whenever they were not in use (i.e. the rationale behind automation was providing comfort to the users); and 3) Persuasive feedback: a combination of realtime ambient feedback and subtle visual hints to support the user's decision-making about when to switch off the appliance were built-in in some coffee machines. The rationale of this latter strategy was based upon some principles of the persuasive design defined by Fogg and Oinas-Kukkonen et al. [10][17]: tunneling, reduction, suggestion, physical attractiveness of the source and cooperation between the physical object and the user to reduce energy consumption.

Because of five out of fifteen groups dropped out the experiment before completion, the assignment of the experimental conditions among remaining groups was as follows: Delayed feedback (3 groups), Automation (3 groups) and Persuasive feedback (4 groups).

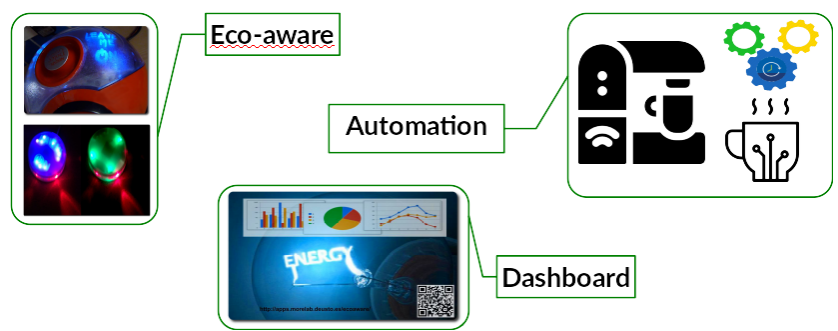

Figure 2. The three different experimental treatments that were randomly assigned to the ten work-groups.

At the end of the experiment, we run several focus group sessions with each of the groups according to Merton et al's. guidelines [16]. The participants of these sessions were workers that volunteered to join the qualitative dynamics $(\mathrm{N}=31)$. We managed to have -at least- one representative from each of the ten remaining groups. Audio data from the

\footnotetext{
${ }^{4}$ Here it is important to remark that we provided collective interaction rather than individual as we were interested in the behaviour of the work teams as a whole.
}

focus group sessions were manually transcribed for further analysis through Grounded Theory methodology.

The study that we conducted demonstrated quantitatively that the the eco-aware treatment helped to save the most of the energy among the three tested. In the following sections, we provide qualitative data from the focus groups as the aim of this paper is to provide design-insights of successful systems.

\section{A. Grounded Theory-based Analysis}

Whilst science has a strong reliance on quantitative and experimental methods, there are many complex, socially based phenomena in HCI that cannot be easily quantified or experimentally manipulated. Within HCI, there is the recognition that the focus on tasks is not enough to design and implement an effective system. Therefore, identifying the users' emotional and social drives and perspectives; their motivations, expectations, trust, identity, social norms and so on is paramount for creating more than 'just appealing' designs [1] (even more if these concepts were related to work practices, communities and organisational social structures).

Borrowing these ideas from Adams et al. [1], we analysed the qualitative data extracted from each of the focus group sessions through Grounded Theory (GT). This qualitative method was applied to produce design insights for creating augmented persuasive devices that promote energy efficiency practices taking into account the social phenomena of the workplace context. GT is an established method for studying qualitative data where codes are generated from the data rather than pre-existing categories. It includes open, axial and selective coding as phases to generate new theory as can be observed in Figure 3.

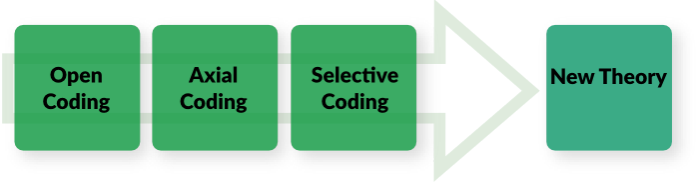

Figure 3. The different phases of Grounded Theory.

The purpose of open coding is to identify entities, to group them into categories, and to describe relevant properties and dimensions pertaining to a category. The codes are extracted iteratively from the data in the process of analysis. Axial coding then identifies relationships between categories as well as conditions, context variables, and resulting consequences. Finally, selective coding is about deliberately setting a focus for the analysis. Thus, the core-category is selected. In the whole process of the GT analysis, interrater reliability of extracting the codes and categories from the focus group data was assessed by two researchers as suggested by Armstrong et al. [2]. 


\section{RESUlts}

In the Open coding phase 132 unitary codes were extracted. Attributes such as frequency, target, intensity or duration were annotated together with each of the emerging codes. Following the constant comparative method [7], saturation of codes and categories was reached in the Axial phase. Five categories emerged from the initial codes: 1) interaction, 2) mediator/emotion, 3) attachment/confidence, 4) context, and 5) behaviour. Finally, the Selective coding phase aims to obtain the central category as mediator for the axial categories. From the analysed data, we elucidate that the relationship between the people (workers) and the augmented appliance to jointly cope with energy inefficiency was the central theme of the theory. Indeed, it brings together the other five axial categories.

Figure 4 shows a diagram interrelating the different categories around the central theme in a linear fashion. According to Charmaz [7], the diagram is devised to organise the incipient theory and to see the relative power, scope, and direction of the categories in the analysis as well as the connections among them for linear narrative purposes. Therefore, the diagram should not be understood as a predictive model.

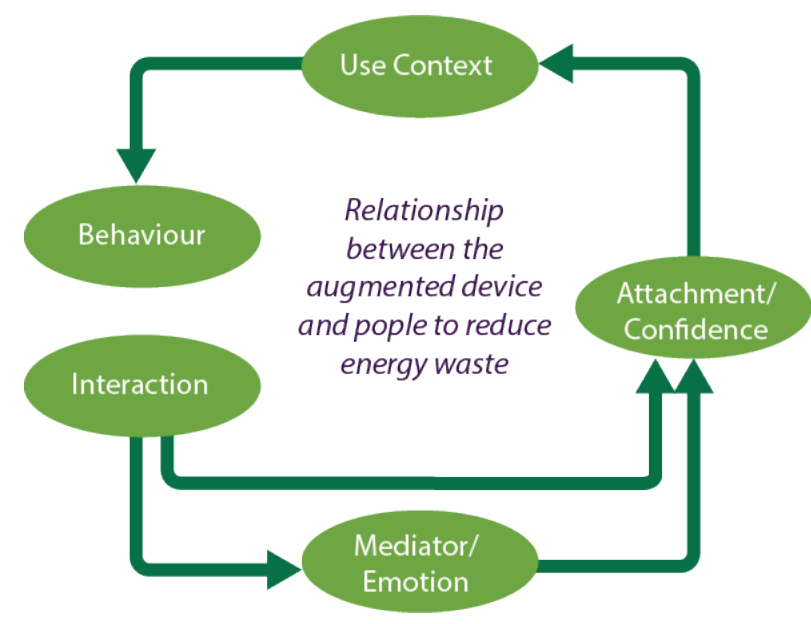

Figure 4. The emerging theory obtained by analysing the qualitative data through Grounded Theory.

\section{AnALYSis AND Design Insights}

In this Section, relevant quotes from participants that interacted with the persuasive coffee maker are reported for each of the five emerging themes. These quotes are jointly offered with a discussion over the implications that the themes have for the design of augmented objects that promote sustainable behaviours.

\section{Interaction}

This category covered one of the most recurrent topics in the focus group sessions and it captured the majority of unitary codes in the GT analysis. Notably, we received positive and pleasant feedback when participants evaluated the interaction with the persuasive coffee maker. However, constructive criticism to improve the proposed design was provided by some people, P1: "I lacked some audible ecofeedback when I put my mug on the augmented appliance's coaster. Something like the NFC/RFID-based readers that one can find in the turnstile of the Metro stations that give you feedback about your pass checking". Surprisingly, some participants that interacted with the persuasive appliance missed some of the aspects that were implemented in other experimental treatments, P2: "I would like to have a Webdashboard where I could track my kWh". The conclusion of this category is that people felt complacent with the feedback received because it was intuitive and helpful to behave in an energy efficient fashion. Design-insight: the designers should be aware that the 'one-size fits all' approach will not fit all needs. Further, in the qualitative phase it is more than probable that some of the interviewees will miss features that the literature reckons that do not work for changing the intended behaviour in the mid and long term. These opinions should be taken with extra care in the designing phase because they may blur the goal of changing the behaviour.

\section{Mediator/Emotion}

Whereas the majority of the feedback provided about the interaction was positive, the proposed persuasive design provoked polarised emotions that were retrieved in the focus group sessions. For example, P3 voiced: "Despite of the suggestions of the augmented appliance to leave it on, I felt cross leaving the coffee maker switched on after preparing a coffee". More positive were the opinions of P4: "I loved to know that we have a smart appliance in the office" or P5: "It is funny to have one [the appliance]...for me it is like it was another member of the department". This latter quote resounds to the 'Media Equation Theory' [20] that states that media and computers are treated by people as they were real humans. Moreover, P5's comment and that of P6: "The coffee-maker sparked conversations about energy consumption among colleagues. Overall during the initial weeks of the set-up" correlated with the idea that augmented smart objects may have the role of mediators to bring about reflections and conversations centred in the topic that the designer wants to change. Design-insight: we want to emphasise the idea that augmented objects seemed to be more than mere relays of eco-feedback, they should be conceived as new interactive actors that may be considered as peers for people to cope with energy inefficiency.

\section{Attachment/Confidence}

As well as the previous category, again two themes were brought together by participants and therefore they are joined into a single category. On the one side, attachment to the smart persuasive appliance was found on several participants 
that were upset when we communicate to them that the smart features were about to be removed, P7: "I will miss the appliance. I changed my perception of it...from being a bare device to considering it as something that is doing well for the environment". On the other side, confidence in the technology was a recurrent theme due to the time that people stay together with the device daily (one year), P8: "I will always heed the eco-coffee machine advices without any doubts...whenever it says 'leave me on', I leave it on without thinking twice". Design-insight: we reckon that in order to strengthen these two themes, which are pivotal for the persuasive process, the augmented everyday object should be designed to remain close to the people to stick influencing throughout the time.

\section{Use Context}

Context of use is defined as "the actual conditions under which a given artifact is used in a normal working situation". According to Pierce et al. [19] there are two important dimensions when designing eco-feedback technologies for use-contexts: dweller control and third party control. The workplace lays into "low dweller control, high third party control". Surprisingly enough, the comments from experiment participants were not only related to the place they work, and therefore, where they use the coffee machine daily. The majority of regressions and opinions were related to the behaviours that one do or learn in one use-context (e.g. at work) and export them to another, maybe different, usecontext (e.g. to home settings - "high dweller control, low third party control"). For example P9 stated: "Because here [at workplace] I switch off the coffee-maker, when I'm at home I pay more attention to energy efficiency". However, care must be taken when designing objects to form new behaviours in a specific context, P10: "Sometimes at home I forget the coffee maker switched off because at work I'm used to leave it on since it [the augmented appliance] pries to me to do so". Design-insight: the persuasive designers should take into account that the spillover effects of the formed behaviour may be beneficial at one use-context but be offering a detrimental consequence in another context.

\section{Behaviour}

This category, along with that of Interaction, was the most popular topic in the conversations that aroused in the focus group sessions. The participants that interacted with the persuasive experimental condition were found to be the most aware of the energy inefficiency issue in the workplace. Moreover, the comments from participants offered initial hints of behaviour change, P11: "We were fully aware of our misleading behaviour at the beginning of the experiment because the coffee maker turned into red due to excessive energy consumption" or P12, "The coffee maker has made me aware of energy consumption. Now...sometimes after preparing a coffee, I take two step back to double check if I've left the appliance on or off". The voices from participants that assured that the newly formed behaviour will remain throughout the time were the most relevant finding, P13: "Somehow I have started form a habit...even if it is something unconscious" or P14: "At the end, we all have got used to switching off the appliance. I believe that we will maintain this easy course of actions". Design-insight: the problem that persuasive designers may find in successful studies (such as the one reported here), is to witness a rebound effect when the experimental conditions are removed by the researcher or are dismissed by participants. Thus, the question we wonder is: what would be the contingency plan if there were no hints or advice to facilitate the decision-making anymore, or, if the rewards finished suddenly, or if the gamification process became annoying because of its repetitiveness, and so on? The designer of augmented everyday objects should carefully plan the longitudinal extent of his/her system and to reflect on the foreseeable consequences of feedback removal.

\section{CONCLUSIONS AND OUTLOOK}

In this article, we have reviewed the state of the art on augmented smart devices for boosting sustainability in a comparative manner through a set of ten well-known strategies borrowed from persuasion and behaviour change theories.

Furthermore, the qualitative data derived from a set of focus groups after carrying out a longitudinal experiment on energy awareness at workplace were analysed using the Grounded Theory approach. GT analysis was applied to produce new insights on the design process of novel persuasive physical interfaces or augmented everyday objects that promote energy-efficient behaviour change at the workplace. The emerging theory presents five categories: 1) interaction, 2) mediator/emotion, 3) attachment/confidence, 4) context, and 5) behaviour. Each of these categories derived from the analyses represents a thematic design-insight, enabling researchers to build effective interventions in the workplace that place in the centre the relationship between people and the augmented device to reduce energy waste (the central category of the emerging theory): from how people will interact with the device, through the emotions which the device may arise, the conversations that the device itself might foster among users, the environment or context where the device will be installed and used, and finally, the behaviour to be promoted. One of the recurrent findings in several categories was the sense of lasting for the augmented device and the behaviour recently formed. Thus, on the one side, we found a tension between the experimental procedure and the desire for participants to retain the augmented device beyond the predefined schedule. Along the same line, people were hesitant when we asked them about whether they thought that their behaviour would have been maintained through the time if the smartness of the appliance was removed. 
Future work will be focused on constantly comparing the initial codes and categories with new sample data obtained from similar longitudinal studies. The aim will be to discover what are the factors of major influence over the energyefficient behaviour among those that already emerged from the theory presented. Moreover, future theoretical sampling should help to better understand whether the model categories 'mediator/emotion' and 'attachment/confidence' extracted from the data will remain united, will be combined into a single category, or conversely, will be separated in four different categories. Finally, further research in different usecontexts beyond the workplace (i.e. low dweller control, high third-party control) should be carried out to test whether the findings derived in the presented model might also apply in them (e.g. public buildings, home settings or dormitories).

\section{ACKNOWLEDGMENTS}

This work has been partially supported by the European Commission through the project HORIZON 2020RESEARCH \& INNOVATION ACTIONS (RIA)-696129GREENSOUL. We gratefully acknowledge the support of the Ministry of Economy, Industry and Competitiveness of Spain under Grant No.: TIN2017-90042-R.

\section{REFERENCES}

[1] Anne Adams, Peter Lunt, and Paul Cairns. A qualititative approach to hci research. In Paul Cairns and Anna Cox, editors, Research Methods for Human-Computer Interaction, pages 138-157. Cambridge University Press, Cambridge, UK, 2008.

[2] David Armstrong, Ann Gosling, John Weinman, and Theresa Marteau. The place of inter-rater reliability in qualitative research: an empirical study. Sociology, 31(3):597-606, 1997.

[3] Ernesto Arroyo, Leonardo Bonanni, and Ted Selker. Waterbot: exploring feedback and persuasive techniques at the sink. In Proc. of CHI'05, pages 631-639. ACM, 2005.

[4] Loove Broms, Cecilia Katzeff, and et al. Coffee maker patterns and the design of energy feedback artefacts. In Proc. of DIS'10, pages 93-102. ACM, 2010.

[5] Hronn Brynjarsdóttir, Maria Håkansson, Eric Baumer, Carl DiSalvo, Phoebe Sengers, and Pierce, James. Sustainably unpersuaded: How persuasion narrows our vision of sustainability. In Proc. of CHI'12, pages 947-956. ACM, 2012.

[6] Diego Casado-Mansilla, Juan López-De-Armentia, Pablo Garaizar, and Diego López-De-Ipiña. To switch the coffee maker or not: That is the question to be energy efficient at work. In Proc. of CHI'14 (E.A.), pages 2425-2430, 2014.

[7] K. Charmaz. Constructing Grounded Theory: A Practical Guide Through Qualitative Analysis. SAGE Publications, 2006.

[8] Benjamin R. Cowan, Chris P. Bowers, Russell Beale, and Charlie Pinder. The stroppy kettle: An intervention to break energy consumption habits. CHI EA '13, pages 1485-1490. ACM, 2013.
[9] R. De Young. Changing behavior and making it stick. Environment and Behavior, 25:485-505, 1993.

[10] B J Fogg. Persuasive Technology: Using Computers to Change What We Think and Do, volume 18. Morgan Kaufmann Publishers, 2003.

[11] Anton Gustafsson and Magnus Gyllenswrd. The power-aware cord: energy awareness through ambient information display. In Extended abstracts of CHI'05, pages 1423-1426. ACM, 2005.

[12] David Holstius, John Kembel, Amy Hurst, Peng-Hui Wan, and Jodi Forlizzi. Infotropism: Living and robotic plants as interactive displays. In Proceedings of the 5th Conference on Designing Interactive Systems: Processes, Practices, Methods, and Techniques, DIS '04, pages 215-221. ACM, 2004.

[13] Li Jönsson, Loove Broms, and Cecilia Katzeff. Watt-lite: energy statistics made tangible. In Proc. of DIS'10, pages 240-243. ACM, 2010.

[14] Karin Kappel and Thomas Grechenig. "show-me": Water consumption at a glance to promote water conservation in the shower. Persuasive '09, pages 1-6. ACM, 2009.

[15] Bran Knowles, Lynne Blair, Stuart Walker, Paul Coulton, Lisa Thomas, and Louise Mullagh. Patterns of persuasion for sustainability. DIS '14, pages 1035-1044. ACM, 2014.

[16] Robert K. Merton, Marjorie Fiske, and Patricia L. Kendall. The focused interview : a manual of problems and procedures (2nd Edition). The Free Press, 1990.

[17] H. Oinas-Kukkonen and M. Harjumaa. Persuasive systems design: Key issues, process model, and system features. Communications of the Association for Information Systems, 24(1), 2009.

[18] J. Pierce and E. Paulos. Beyond energy monitors: Interaction, energy, and emerging energy systems. In Proc. of CHI'12, pages 665-674. ACM, 2012.

[19] James Pierce, William Odom, and Eli Blevis. Energy aware dwelling: a critical survey of interaction design for ecovisualizations. In Proceedings of the 20th Australasian Conference on Computer-Human Interaction: Designing for Habitus and Habitat, pages 1-8. ACM, 2008.

[20] Byron Reeves and Clifford Nass. The Media Equation: How People Treat Computers, Television, and New Media Like Real People and Places. Cambridge University Press, 1996.

[21] A. Thieme, R. Comber, J. Miebach, J. Weeden, N. Kraemer, S. Lawson, and P. Olivier. We've bin watching you": Designing for reflection and social persuasion to promote sustainable lifestyles. In Proc. of CHI'12, pages 2337-2346. ACM, 2012.

[22] S. Wendel. Designing for Behavior Change. O'Reilly Media, 2013. 